\title{
Zum 75. Todestag von Professor Maximilian Perty, Bern (1804-1884) Eine allgemein-persönliche und mikrographische Würdigung
}

\author{
Von Josef Kurmann, Liebefeld-Bern
}

Joseph Anton Maximilian Perty wurde am 17. September 1804 in der bayrischen Provinz Franken geboren, wo sein Vater, aus seinem Heimatlande Ungarn übergesiedelt, die Stelle eines Rechnungsbeamten bekleidete. Gemäß dem Verlangen seiner Eltern studierte er Medizin. Das Ziel, das ihm von Jugend an vorschwebte, war, sich ganz dem Studium der Naturwissenschaften, speziell der Zoologie hinzugeben. Zu diesem Zwecke erwarb er sich neben dem medizinischen noch den philosophischen Doktorgrad in Erlangen und habilitierte sich 1831 in München als Privatdozent für Zoologie und allgemeine Naturgeschichte.

München gehörte damals zu den für Naturforscher besonders anregenden Universitäten Deutschlands. Hier lehrte der Naturphilosoph Schelling neben dem Zoologen OKen. Beide Männer hatten dasselbe Ziel, nämlich die Naturwissenschaften aus den Bahnen der einfachen Naturbeschreibung herauszureißen und die zahlreichen Einzeltatsachen in das Licht philosophischer und allgemeiner Gesichtspunkte zu heben. Waren auch diese Versuche zum Teil verfrüht und die Basis, auf welche das naturphilosophische System gestellt wurde, mannigfach unsicher, so mußte doch die ganze Richtung eine tiefer denkende Natur mächtig ergreifen, besonders wenn ein Mensch wie eben auch Perty einer höheren Auffassung zugänglich war. Man wollte nicht in den Einzelheiten untergehen, sondern das Beobachtete auf allgemeine Prinzipien zurückführen, um über dem Einzelnen das Ganze nicht zu vernachlässigen.

Mit der Gründung der bernischen Hochschule im Jahre 1834 wurde Perty zum ordentlichen Professor für Zoologie und Naturgeschichte ernannt. Er bekleidete diese Professur 41 Jahre. Wegen seines Fleißes nannte man ihn die «Biene» der Hochschule. Als Lehrer entfaltete Perty eine ungewöhnlich mannigfaltige Tätigkeit. Er lehrte Zoologie, allgemeine Naturgeschichte, Geschichte der Naturwissenschaften, Anthropologie, Entomologie, Naturphilosophie, einige Zeit auch Botanik, und in praktischen Kursen unterrichtete er seine Schüler im Gebrauche des Mikroskopes, welches er meisterhaft handhabte. 
Angeregt durch die bahnbrechenden Arbeiten des dänischen Zoologen Müller (1786), des deutschen Zoologen Ehrenberg (1838) und des französischen Zoologen DuJardiv (1841) über die Urtiere untersuchte er die mikroskopischen Lebensformen seiner neuen und geliebten Heimat, der Schweiz. Die Forschungsergebnisse wurden in dem sehr beachteten Werke Zur Kenntnis kleinster Lebensformen, Bern 1852, niedergeschrieben. Dabei ist Perty in seiner Klassifikation der Infusorien von derjenigen seiner Vorgänger vielfach abgewichen und hat einen selbständigen Weg eingeschlagen. Er gibt eine Aufzählung von der in der Schweiz gefundenen Urtiere, wobei zwischen der ersten Klasse der Infusorien mit erster Ordnung der Ciliata (Wimpertierchen) und zweiter Ordnung der Phytozoida (Pflanzentierchen) unterschieden wird. Als zweite Klasse werden die Rhizipoda angeführt. Wie Dujardin in Frankreich und Werneck in Salzburg, so hat Perty in der Schweiz eine Menge Infusorien gefunden, welche vorher unbekannt waren. Perty entdeckte ebenfalls die Schwefelbakterien ${ }^{1}$ und war der erste, der für die Diagnose der Bakteriengattungen entwicklungsgeschichtliche Merkmale verwendete sowie die Sporenbildung bei den Bakterien beobachtete. Er stellte drei neue Bakteriengattungen auf, die, vom systematischen Standpunkt aus gesehen, einen wirklichen Fortschritt bildeten. Perty stellt die Bakterien systematisch zu den Tierchen (Ordnung Phytozoida), läßt aber als erster die Möglichkeit zu, dieselben auch im Pflanzenreich unterzubringen. Neben diesen gründlichen und bedeutenden mikrographischen Untersuchungen entfaltete Perty eine reiche, vielseitige und fruchtbare literarische Tätigkeit.

Nachdem Perty bereits in der entomologischen und ethnologischen Gesellschaft zu London aufgenommen worden war, trug ihm das Werk Die Anthropologie auch den Titel eines Ehrenmitgliedes des anthropologischen Institutes von England und Irland ein. Zudem war er Mitglied der Deutschen Akademie der Naturforscher mit dem Beinamen Oken, des ihm geistesverwandten berühmten deutschen Zoologen, der Bayrischen Akademie der Wissenschaften, der Zoologisch-Botanischen Gesellschaft zu Wien und war schließlich ebenfalls ein sehr eifriges Mitglied der Schweizerischen Naturforschenden Gesellschaft.

In den Jahren 1837 bis 1846 erschien das vierbändige Werk Allgemeine Naturgeschichte als philosophische und Humanitätswissenschaft, in welchem er versuchte, im Sinne Okens die gesamte Naturgeschichte vom philoso-

1 Mitteilung von Herrn Professor Schopfer. 
phischen Gesichtspunkt aus darzulegen. Aber alle diese Studien waren für seinen Geist nur das Mittel zu höherem Zweck. Er wollte nicht nur Tatsachen sammeln, sondern, gestützt auf diese, den Geheimnissen des Lebens und der Menschenseele nachforschen, das war und blieb sein Ziel.

Schon in seiner Jugend hatte er seine Vorliebe für die Zoologie damit begründet, daß er sie als die Wissenschaft bezeichnete, welche zum Menschen führt. Wir können seine Neigung, allgemeine Gesichtspunkte anzustreben, nicht besser charakterisieren, als wenn wir seine eigenen. Worte anführen, welche in der Vorrede zum dritten Band seiner Allgemeinen Naturgeschichte niedergelegt sind: «Die sogenannte Naturgeschichte wird ihren Namen erst verdienen, wenn ihre Gestaltung ihr entspricht. Ist die Beschreibung einer Sammlung von Statuen eine Kunstgeschichte? Ebensowenig als die Beschreibung einer Reihe von Naturkörpern schon Naturgeschichte ist. Soll ein Werk diesen Namen verdienen, so muß es in den Plan des schaffenden Geistes selbst eindringen und den Gang seiner Gedanken verfolgen. Wie der wahre Geschichtsforscher nicht bloß die Tatsachen aufzählt, sondern auch deren Genesis ergründet, so muß auch der wahre Naturforscher den Quellen nachspüren, aus welchen die gegenwärtige Natur als eine fertige Erscheinung entsprungen ist und den Zusammenhang alles Einzelne zu erkennen suchen. » - In diesem Streben leitete ihn erst die Metaphysik, dann die Psychologie, wie sie in der Betrachtung des Tieres und im Völkerleben sich offenbart. Wir verdanken dem Studium Pertys in letzterer Richtung seine beiden Werke Über das Seelenleben der Tiere und Die Anthropologie als die Wissenschaft von dem körperlichen und geistigen Wesen des Menschen.

Bei großem Lehreifer, bei nicht seltenen Lichtpunkten, die für ihn die besondere Strebsamkeit und Anhänglichkeit einzelner Schüler bildeten, war er doch viel zu sehr eine stille Gelehrtennatur, als daß er zu einer äußerlich glänzenden akademischen Wirksamkeit hätte gelangen können. Mit Pertys Veranlagung und Betätigung in der Naturwissenschaft, Geschichte, Psychologie, Philosophie und Mystik hatte er für einen Naturforscher des 19. Jahrhunderts eine seltsame Mischung geistiger Elemente, welche mit einer wohltuenden Einheit der Persönlichkeit verbunden waren. Seine Pflichttreue, Arbeitsliebe, sein idealer Sinn, Fleiß und Streben nach Wahrheit werden uns immer ein Vorbild sein. 
Anhang: Verzeichnis der bekanntesten Schriften von Professor Maximilian Perty

1. Perty M., Delectus Animalium articulatorum Brasiliae, Jahr?

2. Perty M., Mikroskopische Präparate aus dem Pflanzenreich, in Das mikroskopische Institut von Enzell und Comp., 5. Heft, 4. Lieferung, Zürich, Jahr?

3. Perty M., Observationes nonnullae in Coleoptera Indiae orientalis, München 1831.

4. Perty M., De Insectorum in America meridionali habitantium vitae genere... Observationes nonnullae.

5. Perty M., Über die höhere Bedeutung der Naturwissenschaften und ihren Standpunkt in unserer Zeit. Eine akademische Eröffnungsrede. Bern 1835.

6. Perty M., Rede zur Jahresfeier der Eröffnung der Hochschule in Bern, den 15. November 1837.

7. Perty M., Allgemeine Naturgeschichte, 3 Bände, Bern 1837-1841.

8. Perty M., Allgemeine Naturgeschichte, Bern 1843-1846, I-IV (4 Bände).

9. Perty M., Über den Begriff des Tieres und die Einteilung der tierischen belebten Wesen, Taf., Bern 184.6.

10. Perty M., Die Gymnasien als Bildungsanstalten für den Humanismus im weiteren Sinn, Bern 1846.

11. Perty M., Die Bewegung durch schwingende mikroskopische Organe im Tier- und Pflanzenreiche. Nebst Erörterung über Sporozoiden, Infusorien, Bacillarien und über die Elementarstruktur der Halcyonella fluviatilis var. Nymphaeae, Bern 1848.

12. Perty M., Zur Kenntnis kleinster Lebensformen nebst Spezialverzeichnis der in der Schweiz beobachteten, Bern 1852.

13. Perty M., Über die Bedeutung der Anthropologie für Naturwissenschaft und Philosophie. Ein Vortrag gehalten vor der Schweiz. Naturforschenden Gesellschaft in Sitten, den 19. August 1852, Bern 1853.

14. Perty M., Vorschule der Naturwissenschaft, Stuttgart 1853 (Praktische Lehrbücher, Band 4).

15. Perty M., Die Zoologie: 1. Allgemeine Zoologie von Agassiz und Gould. 2. Spezielle Zoologie von Perty, Stuttgart 1855.

16. Perty M., Über die Seele, Bern 1856.

17. Perty M., Grundzüge der Ethnographie, Leipzig und Heidelberg 1859.

18. Perty M., Die mystischen Erscheinungen der menschlichen Natur, Leipzig und Heidelberg 1861.

19. Perty M., Die Realität magischer Kräfte. Wirkungen des Menschen, Leipzig und Heidelberg 1863.

20. Perty M., Anthropologische Vorträge gehalten im Winter 1862-1863 in der Aula zu Bern, Leipzig und Heidelberg 1863.

21. Perty M., Einige Insekten-Mißbildungen, in Mitteilungen der Naturforschenden Gesellschaft Bern 1866. 
22. Perty M., Blicke in das verborgene Leben des Menschengeistes, Leipzig und Heidelberg 1869.

23. Perty M., Die Natur im Lichte philosophischer Anschauung, Leipzig und Heidelberg 1869.

24. Perty M., Über den Parasitismus in der organischen Natur, Berlin 1869 (Sammlung gemeinverständlicher wissenschaftlicher Vorträge Ser. IV, H. 91).

25. Perty M., Die Anthropologie als die Wissenschaft von dem körperlichen und geistigen Wesen des Menschen, Band 1 und 2, Leipzig und Heidelberg 1874.

26. Perty M., Über die Grenzen der sichtbaren Schöpfung nach der jetzigen Wirkung der Mikroskope und Fernrohre, Berlin 1874 (Sammlung gemeinverständlicher wissenschaftlicher Vorträge, Ser. IV, H. 195).

27. Perty M., Über das Seelenleben der Tiere, 2. Auflage, Leipzig und Heidelberg 1876.

28. Perty M., Der jetzige Spiritualismus und verwandte Erfahrungen, Leipzig und Heidelberg 1871.

29. Perty M., Erinnerungen aus dem Leben eines Natur- und Seelenforschers des 19. Jahrhunderts (Portrait), Leipzig 1879.

30. Perty M., Die sichtbare und unsichtbare Welt, Leipzig und Heidelberg 1881.

31. Perty M., Ohne die mystischen Tatsachen keine erschöpfende Psychologie, Leipzig und Heidelberg 1883.

Dieses Verzeichnis erhebt nicht Anspruch auf Vollständigkeit. Weitere Angaben über die Persönlichkeit von Professor Maximilian Perty sind in der Grabrede, gehalten von Professor Theophil Studer, Bern, gedruckt im Bund, Jahrgang 1884, enthalten. 